\title{
Robot-assisted techniques in vascular and endovascular surgery
}

\author{
A. Püschel ${ }^{1}$ (D) C. Schafmayer ${ }^{1} \cdot$ J. Groß $^{1}$
}

Received: 11 August 2021 / Accepted: 9 February 2022 / Published online: 28 February 2022

(c) The Author(s), under exclusive licence to Springer-Verlag GmbH Germany, part of Springer Nature 2022

\begin{abstract}
For thousands of years, robots have inspired the imagination of humans, but it was only about 35 years ago that a robot was used for the first time in medicine. Since then, robot-assisted procedures have become increasingly popular in urology, general surgical specialties, and gynecology. Robot-assisted vascular surgery was first introduced in 2002 and was thought to overcome the limitations of laparoscopy. However, it did not gain widespread popularity, and its usage is still limited to a few centers worldwide. Robot-assisted endovascular procedures, on the other hand, while still in its infancy, have become a promising alternative to existing techniques. The improvements of the robotic systems promote better surgical performance and reduce occupational hazards for vascular and endovascular surgeons. A comprehensive review of literature was performed using the search terms "robotic," "robot assisted," "vascular surgery," and "aortic" for surgical procedures or "robotic," "robot assisted," and "endovascular" for endovascular procedures. Full text articles that were published between January 1990 and March 2021 were included. This review summarizes the development of the techniques for robot-assisted vascular and endovascular surgery in recent years, its outcomes, advantages, disadvantages, and perspectives.
\end{abstract}

Keywords Robotic $\cdot$ Robotic-assisted · Vascular surgery $\cdot$ Endovascular $\cdot$ Intervention

The whole field of surgery is developing towards minimal invasiveness, and robotic surgery has become increasingly popular in many fields.

The first surgical application of robotic technology was used in 1985 by Kwoh et al. to undertake stereotactic brain biopsy with the PUMA 560 [1]. Early active robotic systems demonstrated the potential of mechanical devices to enhance surgical procedures. The driving force, however, for the development of the contemporary platforms was the concept of telepresence initially introduced by a collaboration between the NASA Ames Research Center and researchers from Stanford with support by the US military.

Further development of robotic systems was carried out by Computer Motion with the introduction of the AESOP (automatic endoscopic system for optimal positioning) robotic platform. Modifications resulted in the ZEUS robotic system that was FDA approved in 2001. Intuitive Surgical released a robotic platform that was later to become the da

A. Püschel

anja.pueschel@med.uni-rostock.de

1 Universitätsmedizin Rostock, Klinik für Allgemein-, Viszeral-, Gefäß- Und Transplantationschirurgie, Schillingallee 35, 18057 Rostock, Germany
Vinci system. It received its first FDA approval for general laparoscopic surgery in 2000. In 2003 Computer Motion and Intuitive Surgical merged, and the ZEUS and da Vinci systems were unified $[2,3]$.

Since then, the da Vinci system has been applied in different specialties like urology, colorectal surgery, and gynecology. In vascular surgery, it was first used in 2002 in robot-assisted laparoscopic aorto-bifemoral bypass surgery [4]. Over the years, feasibility for robotic vascular surgery has been demonstrated in a variety of laparoscopic vascular reconstructions, mainly of the aortoiliac and visceral arteries, and only recently, robot-assisted endovascular procedures have become a promising alternative to existing endovascular techniques.

In 2020, approximately $1,243,000$ robotic procedures were performed worldwide with Da Vinci Surgical Systems. This is an increase to 2019 and 2018 with 1,229,000 and $1,038,000$ procedures, respectively, despite all difficulties caused by the COVID-19 pandemic [5, 6].

The da Vinci system, as most surgical robots, is actually a telemanipulator as it lacks the autonomous elements of a robot and is entirely dependent on human activity. It is a master-slave system that consists of a patient side cart with three to four interactive arms and the surgeon's console for 
remote manipulation. Compared with conventional laparoscopy, there are several technological improvements such as enhanced visualization due to the 3-dimensional interface, improved wrist motion freedom, motion scaling, tremor filtration, and improved ergonomics. An interesting future development is the creation of "virtual fixtures," using software to create "no-go" areas and preventing robotic instruments from entering and damaging sensitive tissues, e.g., blood vessels [7]. Additionally, this technology gives surgeons the ability to perform telesurgery, the reason for its initial development [8].

There are, however, some disadvantages to this innovative robotic system. First, larger operating rooms to accommodate the whole system are required. Additionally, staff needs to be trained, and there are no haptic sensors. Although robotic surgeons compensate for it by becoming more susceptible to visual cues, its lack of haptic feedback still may lead to increased operating times and higher learning curve and may cause tissue damage [9].

The main issue, however, are the higher costs when compared to conventional approaches. The costs for the system are approximately $\$ 1.5$ million for each unit, with annual service costs of approximately $\$ 112.000$ per year and extra cost for disposable supply [10]. Cost comparisons between conventional and robotic-assisted procedures vary from hospital to hospital and between the health systems. But with increasing surgeons' experience, costs may be reduced [11].

For this review, a comprehensive search of literature was performed on PubMed using the terms "robotic" OR "robotassisted" AND "vascular surgery" OR "aortic" for surgical procedures or "robotic" OR "robot-assisted" AND "endovascular" for endovascular procedures resulting in 884 and 330 results, respectively. The search timeline ranged from January 1990 to March 2021. Only clinical studies reported in English were included. Animal or ex vivo experimental studies were excluded. Additionally, PubMed listed articles from the authors' personal archives were explored and the information included in this article.

\section{Robot-assisted (laparoscopic) vascular surgery}

Laparoscopic surgery leads to faster recovery by reducing operative trauma. In vascular surgery, especially aortic repair, it combines minimally invasiveness with durable results of conventional surgery [12]. The first laparoscopic vascular surgery was performed in 1993 as a laparoscopically assisted aorto-bifemoral bypass [13]. Since then, an increasing number of patients was successfully treated, and new techniques and approaches have been developed. These include totally laparoscopic as well as laparoscopically assisted and laparoscopically hand-assisted techniques.
However, only a small number of clinical studies have been published, even fewer series on laparoscopic abdominal aortic aneurysm repair. It appears to be more difficult than bypass surgery and competes against endovascular aneurysm repair (EVAR) as a minimally invasive technique with a low morbidity and mortality [12]. In light of the rapid development of endovascular procedures, laparoscopic aortic surgery did not gain widespread popularity, mainly due to technical challenges, difficulty acquiring the necessary skills, especially for not laparoscopically experienced vascular surgeons, and hence a long operating time.

Robot-assisted (RA) laparoscopic surgery, on the other hand, is another possible alternative for minimally invasive vascular surgery. It overcomes the limitations of laparoscopy and results in a higher level of precision and control in confined spaces as well as in a shorter learning curve [14].

Laparoscopic or RA-(laparoscopic) surgery can be as less invasive alternative for aneurysm repair and for bypass surgery for aortoiliac occlusive disease (AIOD) or for secondary interventions after EVAR.

Robot-assisted laparoscopy was first introduced to vascular surgery in 2003 by Wisselink et al. performing an aorto-bifemoral bypass [4]. From 2003 to 2016, a couple of case series from few centers worldwide reported results of robot-assisted laparoscopic surgery in the aortoiliac region. An overview over the largest ones with total robot-ssisted aortic anastomosis is summarized in Table 1.

The conversion rate ranged from 1.3 to $25 \%$, morbidity rates from 0 to $20 \%$ and mortality rates from 0 to $3.5 \%$. High-volume centers tend to have a better outcome.

Stadler et al. reported an improvement of precision, control, and dexterity of the procedure with a high technical success rate. Aortoiliac anastomoses seem to be more accurate and quicker than with conventional laparoscopic techniques $[18,19]$. Contrary to that, other authors have found no improvement of laparoscopic performance or shortening of the learning curve with robotic assistance [20,21].

The total operating time, however, is longer in total robotassisted procedures, as shown by Lin et al. and Kolvenbach et al., mainly due to the technical complexity of the robotic device $[15,18]$.

Besides infrarenal aortic pathologies, robotic devices have been used in other reconstructive arterial procedures such as renal and splenic artery aneurysm repair [22, 23]. Other pathologies that were treated were complications of endovascular procedures such as a persistent type II endoleak after EVAR by robotic ligation of the inferior mesenteric artery and hybrid surgical debranching and endovascular repair of thoraco-abdominal aortic aneurysms (TAAA), but overall numbers were small $[24,25]$.

There are however conflicting results regarding the allover clinical benefits of robot-assisted laparoscopic procedures. Several publications have reported that robotic 
Table 1 Overview of case series of RA-aortoiliac procedures with RA-aortic anastomosis

\begin{tabular}{|c|c|c|c|c|c|c|c|c|c|}
\hline & Year & Robotic system & $\begin{array}{l}\text { Patients } \\
\text { (num- } \\
\text { ber) }\end{array}$ & Operation & $\begin{array}{l}\text { Conver- } \\
\text { sion (num- } \\
\text { ber) }\end{array}$ & $\begin{array}{l}\text { Operating time } \\
\text { (min) }\end{array}$ & $\begin{array}{l}\text { Clamping time } \\
\text { (min) }\end{array}$ & $\begin{array}{l}\text { Morbidity } \\
\text { (number) }\end{array}$ & $\begin{array}{l}\text { Mortality } \\
\text { (number) }\end{array}$ \\
\hline $\begin{array}{l}\text { Kolvenbach et } \\
\text { al. [15] }\end{array}$ & 2003 & Zeus & 10 & $10 \mathrm{AAA}$ & $2(20 \%)$ & $242 \pm 40.5$ & $96 \pm 21.6$ & $1(10 \%)$ & 0 \\
\hline $\begin{array}{l}\text { Desgranges et } \\
\text { al. [16] }\end{array}$ & 2004 & Da Vinci & 5 & $5 \mathrm{AIOD}^{*}$ & $1(25 \%)$ & 188 (mean) & $75 \pm 28$ & $1(20 \%)$ & 0 \\
\hline $\begin{array}{l}\text { Jongkind et al. } \\
\text { [17] }\end{array}$ & 2011 & $\begin{array}{l}5 \text { Zeus } 23 \mathrm{Da} \\
\text { Vinci }\end{array}$ & 28 & 28 AIOD & $4(14 \%)$ & 350 (median) & 70 (median) & $4(14 \%)$ & $1(3,5 \%)$ \\
\hline \multirow{2}{*}{$\begin{array}{l}\text { Stadler et al. } \\
\text { [14] }\end{array}$} & 2016 & Da Vinci & 285 & 61 AAA & $8(13 \%)$ & 253 (median) & 93 (median) & $0(0 \%)$ & $1(1,6 \%)$ \\
\hline & & & & 224 AIOD & $2(0,9 \%)$ & 194 (median) & 37 (median) & $3(1,33 \%)$ & 0 \\
\hline \multirow[t]{3}{*}{ Lin et al. [18] } & 2012 & Da Vinci & 3 & 3 AIOD & $0(0 \%)$ & $494 \pm 36$ & $60 \pm 21$ & & \\
\hline & & & & $9 \mathrm{AIOD}^{* *}$ & $0(0 \%)$ & $425 \pm 94$ & $102 \pm 93$ & N.R. ${ }^{\#}$ & 0 \\
\hline & & & & $7 \mathrm{AAA}^{* *}$ & $1(14 \%)$ & $396 \pm 146$ & $87 \pm 48$ & & \\
\hline
\end{tabular}

AAA, abdominal aortic aneurysm; AIOD, aorto-iliac occlusive disease. *Median-laparotomy in 3 patients; **a mini-incision was used for clamping, \#no detailed attribution to procedures

surgery equals or even improves outcomes by laparoscopic surgery in different specialties [26]. Surgeons worldwide have proved its feasibility and safety for the performance of many different robot-assisted surgeries and reported that it equals outcomes by laparoscopic surgery.

For procedures in confined spaces such as colorectal and esophageal surgery, the robotic system has been shown to be more beneficial $[27,28]$. In bariatric surgery, however, a meta-analysis comparing robotic and conventional laparoscopic gastric bypass did not find differences in mortality but increased operative times [29]. A Cochrane database review from 2017 concluded that there is no high-quality evidence for improved oncological outcomes, postoperative complication rates, and postoperative pain in RA-assisted prostatectomy compared to laparoscopic or open surgery [30]. A controlled randomized multicenter study on RA-laparoscopic versus conventional laparoscopic surgery of rectal cancer reported similar results [31].

So far, good-quality data from randomized trials in vascular surgery is missing as it is limited to individual case studies from few centers worldwide. One reason for this may be that the da Vinci system is not approved for this medical field. An exception is the group of Stadler et al. with the biggest case series of 285 procedures an probably the broadest experience in this regard.

As mentioned before, robotic surgery has its pitfalls, mainly in being more expensive than conventional procedures, and there is still a learning curve, limiting its use to few centers worldwide. With the competition of a rising endovascular field, robot-assisted laparoscopic procedures did not assert themselves in vascular surgery. However, they can still add to the therapeutic options.

\section{Robot-assisted endovascular surgery}

Compared to open procedures for the treatment of aortoiliac occlusive disease, endovascular procedures have superior short-term clinical and economic outcomes [32]. They are minimally invasive, more suitable to high-risk patients, and multiple procedures can be performed during the same operation.

Experience with robotic-controlled catheter systems was initially obtained in cardiology for robotic cardiac ablation and mapping for arrhythmias [33, 34]. The first pilot study of robotic-assisted percutaneous coronary intervention was initially described by Beyar et al. [35].

Since then, the system has undergone multiple refinements, and robotic PCI has been shown to have comparable safety and efficacy to the manual approach [36].

One major benefit of remote vascular intervention is the significant reduction of radiation and radiation-associated occupational hazards such as cancer-related risks, cataract, and atherosclerosis [37, 38]. In the PRECISE study, Weisz et al. demonstrated a median radiation reduction of $95.2 \%$ compared to traditional interventions [36].

Two main types of endovascular interventional robots have initially been used: an electromechanical-based, such as the Sensei robotic navigation system (Hansen Medical), and the magnetically controlled systems, such as the Niobe magnetic navigation system (Stereotaxis St. Louis).

Later Hansen and Philipps developed the Magellan Robotic system - the first purely vascular robot that received FDA approval in 2012. Its production, however, was stopped in 2016.

Corindus Vascular Robotics launched the CorPath200 in 2012 for procedures in the whole cardiovascular system. It 
was first FDA-approved for percutaneous coronary interventions (PCIs). Its successor, the CorPath GRX received FDA clearance for PCIs in 2016 and for peripheral artery disease (PAD) in 2018.

The CorPath is a two-component master and slave system consisting of a remote workspace and a table-side robotic unit. The remote workspace is basically a radiation-shielded mobile workstation that allows independent manipulation of guidewires and catheters, whereas the table-side robotic unit consists of an articulating arm and a robotic drive that houses a single-use cassette which moves the guidewires and rapid exchange catheters.

This endovascular robotic platform enables control of all three interventional devices, i.e., guidewire, catheter, and balloon/stent catheters, in ways that are not possible manually. Rotations in 30 degree increments can be performed and the catheter can be advanced or retracted in 1-mm increments thus enabling exact steerability and precise positioning that helps to maintain the wire and catheter in the center of the vessel lumen and avoids vessel trauma. However, as it is not compatible to all devices, intermittent manual intervention might become necessary [39].

Since its introduction, the endovascular robotic approach has been increasingly used for aortic, peripheral vascular, and neurovascular interventions. An overview of the largest studies is summarized in Table 2.

In 2009, an in vitro study evaluated the role of robotic endovascular techniques in fenestrated grafts [49]. The first robotic-assisted endovascular EVAR was successfully performed by the same group in a 78-year-old patient [50].

But up to date, in vivo experience in robotic-assisted endovascular aortic repair is limited to aortic arch catheter placement during TEVAR [43], contralateral gate cannulation in EVAR [51], and cannulations of renal or mesenteric arteries during FEVAR [52]. Manual intervention is still necessary, but with future technical progress and by combining the robot with three-dimensional fusion imaging technology, these challenging procedures may be performed with little radiation and contrast use, thus significantly improving patients' safety as well as the surgeon's radiation exposure.

In 2016, Mahmud et al. demonstrated for the first time the feasibility and safety of a robotic-assisted peripheral vascular platform for femoropopliteal vessels. A total of 29 lesions in 20 patients with Rutherford category II-III and lesions mainly in the SFA (lesion length $33 \mathrm{~mm} \pm 15,5 \mathrm{~mm}$ ) were successfully treated robot-assisted endovascular with balloon angioplasty. The same group reported successful RA angioplasty in slightly longer lesions $(49 \mathrm{~mm} \pm 37,5 \mathrm{~mm})$ in patients with Rutherford category III-IV [42, 44].

The procedure times as well as fluoroscopy times were comparable to the conventional endovascular therapy. The success of below-the-knee revascularization has so far only been described by one author [53].

Robotic assistance can be of great advantage in challenging anatomic conditions such as severely angulated aortic arches and tortuous carotid arteries that may pose an increased risk for thromboembolism from catheter manipulation.

With endovascular robotic systems, catheters and wires can be kept in the center of the vessel lumen.

A study by Perera et al. found significantly less microembolization in intraoperative transcranial Doppler during robotic catheter placement in the aortic arch compared to manual techniques [43].

The feasibility and safety of carotid artery stenting (CAS) have been demonstrated by several authors for patients with symptomatic [39] and asymptomatic carotid artery stenosis [45].

The first results of RA-endovascular procedures are very promising. However robotic endovascular navigation

Table 2 Overview of cases/case series of clinical applications of robotic endovascular procedures

\begin{tabular}{lllll}
\hline Author & Year & Robotic system & Patients/vessels & Procedure \\
\hline $\begin{array}{l}\text { Bismuth } \text { et al. [40] } \\
\text { Cochennec } \text { et al. [41] }\end{array}$ & 2013 & Hansen & 20 vessels & Iliac artery and SFA cannulation \\
& 2015 & Magellan & 37 vessels & $\begin{array}{c}\text { Visceral and renal vessel cannulation during } \\
\text { FEVAR/BEVAR } \\
\text { Mahmud } \text { et al. [42] }\end{array}$ \\
Perera et al. [43] & 2016 & CorPath 200 & 20 patients & Percutaneous angioplasty of the SFA \\
Cheung et al & 2017 & Magellan & 11 patients & Catheter placement in aortic arch during TEVAR \\
Mahmud et al. [44] & 2020 & Magellan & 14 patients & EVAR gate cannulation \\
Sajja et al. [45] & 2020 & CorPath GRX & 20 patients & Percutaneous angioplasty of the SFA \\
& & CorPath GRX & 7 patients & Cerebral Angiography \\
Weinberg et al. [46] & 2020 & CorPath GRX & 3 patients & Carotid artery angioplasty \\
Nogueira et al. [39] & 2020 & CorPath GRX & 4 patients & Carotid artery angioplasty \\
Desai et al. [47] & 2021 & CorPath GRX & 6 patients & Carotid artery angioplasty \\
Jones et al. [48] & 2021 & Magellan & Cerebral Angiography \\
\hline
\end{tabular}


has still some drawbacks. There is a setup time for the system before each procedure and additional staff training is necessary. But as several in vitro experiments have demonstrated, robotic endovascular surgery is much easier to learn compared to conventional endovascular procedures $[49,54]$.

As in RA- laparoscopic surgery, endovascular robotic systems lack haptic control and thus might risk vascular injury. At present, the endovascular robotic platforms are not compatible to all materials such as guidewires and some devices need to be deployed manually $[39,45]$.

The higher costs for the robotic system and for the disposable cassette also need to be considered; however, the long-term health benefits for the staff and the patients' safety have to be taken into account.

Compared to conventional methods, the advantages of robotic endovascular surgery are shorter procedure time and fluoroscopic exposure time, better stability of the catheter tip, and improved control over catheter movements. After reaching the target site, the system is extremely stable to make submillimeter movements [45].

Further research will be needed to determine whether endovascular robotic systems truly improve patient outcomes and to evaluate the cost-effectiveness and the safety in complex clinical cases.

\section{Telesurgery}

The initial goal of developing robotic surgical platforms was the possibility of performing remote surgical procedures. This is especially imminent as the geographic distribution of highly specialized healthcare limits medical access to a significant proportion of people.

Telesurgery uses wireless networks and robotic technology to connect surgeons and patients that are distantly located from another. The world's first telesurgery, named "Operation Lindbergh," was conducted in 2001 between a team of French surgeons in New York, USA, and a female patient in Strasbourg, France, using a ZEUS robotic system [55]. Further clinical studies have demonstrated its feasibility in interventional cardiology in vivo and in vitro in interventional vascular surgery so far [56-58].

Telerobotic procedures are presently, however, far from being fully operational and more experimental in character. The major challenges are the latency time, delaying audible and visual signals, thus resulting in surgical inaccuracy and a risk of the patient's safety. A stable high-speed-data connection is necessary throughout the procedure [56]. Furthermore, financial and legal issues of remote surgical procedures between different medical centers have to be taken into consideration.

\section{Conclusion}

Robotic technology may enhance surgery by extending human capabilities. With the help of a robotic system, surgeons' movements can be scaled into micromotions, physical tremor is eliminated and, vision is improved, thus facilitating actions that are not possible in conventional surgery.

Robotic surgery has been applied in a variety of laparoscopic vascular reconstructions, such as iliac arteries, visceral arteries, and the abdominal aorta. But most of these procedures were performed solely at a few centers worldwide, and large series have only been published by the most active centers [14].

One of the reasons why it has not been widely adopted in the vascular community is the existence of a broad, well-established endovascular field. In cases where endovascular treatment fails or in complex aortic disease treatment as hybrid procedures it may, however, still provide a minimal-invasive surgical alternative.

In endovascular surgery, on the other hand, a transformation is underway. The utility of endovascular robotics has been proven in PAD, CAS, FEVAR, transfemoral renal, and mesenteric interventions even in challenging anatomic situations [41, 42, 44, 45, 48, 52, 53, 59].

Clinical trials have shown that robotic peripheral vascular interventions are feasible, safe, and reduce patient and operator hazards, such as radiation time.

Further technological improvements are necessary to fully integrate that promising new technology into the clinical setting including advancements in steerability and haptic feedback as well as providing compatibility to existing devices. The future of this technology includes its use for remotely performed procedures such as stroke thrombectomies.

Yet, some limitations remain. To date, only a small number of observational studies or case reports in a few centers worldwide have been published demonstrating the feasibility and safety of robot-assisted endovascular procedures. A valid evaluation of its long-term superiority to conventional endovascular techniques is not possible due to a lack of controlled randomized trials.

The main issue, however, is the costs. Robotic surgery is more expensive than conventional therapies, but in endovascular robotics, higher costs come with considerable health benefits for staff and patients.

\section{Declarations}

Conflict of interest The authors declare no competing interests.

Ethics approval This article does not contain any studies with human participants or animals performed by any of the authors. 


\section{References}

1. Kwoh YS, Hou J, Jonckheere EA, Hayati S (1988) A robot with improved absolute positioning accuracy for CT guided stereotactic brain surgery. IEEE Trans Biomed Eng 35:153-160

2. Lane T (2018) A short history of robotic surgery. The Annals of The Royal College of Surgeons of England 100:5-7

3. Hockstein NG, Gourin CG, Faust RA, Terris DJ (2007) A history of robots: from science fiction to surgical robotics. J Robot Surg $1: 113-118$

4. Wisselink W, Cuesta MA, Gracia C, Rauwerda JA (2002) Robotassisted laparoscopic aortobifemoral bypass for aortoiliac occlusive disease: a report of two cases. J Vasc Surg 36:1079-1082

5. (2021) Intuitive surgical fourth quarter and full year 2019 results

6. (2020) Intuitive surgical fourth quarter and full year 2020 results

7. Brodie A, Vasdev N (2018) The future of robotic surgery. The Annals of The Royal College of Surgeons of England 100:4-13

8. Peters BS, Armijo PR, Krause C et al (2018) Review of emerging surgical robotic technology. Surg Endosc 32:1636-1655

9. Jara RD, Guerrón AD, Portenier D (2020) Complications of robotic surgery. Surg Clin North Am 100:461-468

10. Steinberg PL, Merguerian PA, Bihrle W et al (2008) A da Vinci robot system can make sense for a mature laparoscopic prostatectomy program. JSLS 12:9-12

11. Morelli L, di Franco G, Lorenzoni V et al (2019) Structured cost analysis of robotic TME resection for rectal cancer: a comparison between the da Vinci Si and Xi in a single surgeon's experience. Surg Endosc 33:1858-1869

12. Nio D, Diks J, Bemelman WA et al (2007) Laparoscopic vascular surgery: a systematic review. Eur J Vasc Endovasc Surg 33:263-271

13. Dion YM, Kathouda N, Rouleau C, Aucoin A (1993) Laparoscopy-assisted aortobifemoral bypass. Surg Laparosc Endosc 3:425-429

14. S Stádler P, Dvořáček L, Vitásek $P$, Matouš $P$ (2016) Robot assisted aortic and Non-aortic vascular operations. Eur J Vasc Endovasc Surg 52:22-28

15. Kolvenbach R, Schwierz E, Wasilljew S et al (2004) Total laparoscopically and robotically assisted aortic aneurysm surgery: a critical evaluation. J Vasc Surg 39:771-776

16. Desgranges P, Bourriez A, Javerliat I et al (2004) Robotically assisted aorto-femoral bypass grafting: lessons learned from our initial experience. Eur J Vasc Endovasc Surg 27:507-511

17. Jongkind V, Diks J, Yeung KK, et al (2011) Mid-term results of robot-assisted laparoscopic surgery for aortoiliac occlusive disease. Vascular 19

18. Lin JC, Kaul SA, Bhandari A et al (2012) Robotic-assisted aortic surgery with and without minilaparotomy for complicated occlusive disease and aneurysm. J Vasc Surg 55:16-22

19. Štádler P, Matouš P, Vitásek P, Špaček M (2006) Robot-assisted aortoiliac reconstruction: a review of 30 cases. J Vasc Surg 44:915-919

20. Nio D, Bemelman WA, Balm R, Legemate DA (2005) Laparoscopic vascular anastomoses: does robotic (Zeus-Aesop) assistance help to overcome the learning curve? Surg Endosc 19:1071-1076

21. Nio D, Balm R, Maartense S et al (2004) The efficacy of robotassisted versus conventional laparoscopic vascular anastomoses in an experimental model. Eur J Vasc Endovasc Surg 27:283-286

22. Luke P, Knudsen BE, Nguan CY et al (2006) Robot-assisted laparoscopic renal artery aneurysm reconstruction. J Vasc Surg 44:651-653

23. Marone EM, Peri A, Argenti F et al (2020) Robotic treatment of complex splenic artery aneurysms with deep hilar location: technical insights and midterm results. Ann Vasc Surg 68:50-56
24. Wahlgren CM, Skelly C, Shalhav A, Bassiouny H (2008) Hybrid laparorobotic debranching and endovascular repair of thoracoabdominal aortic aneurysm. Ann Vasc Surg 22:285-289

25. Lin JC, Eun D, Shrivastava A et al (2009) Total robotic ligation of inferior mesenteric artery for type II endoleak after endovascular aneurysm repair. Ann Vasc Surg 23:255.e19-255.e21

26. Prete FP, Pezzolla A, Prete F et al (2018) Robotic versus laparoscopic minimally invasive surgery for rectal cancer. Ann Surg 267:1034-1046

27. Matsuyama T, Kinugasa Y, Nakajima Y, Kojima K (2018) Robotic-assisted surgery for rectal cancer: current state and future perspective. Annals of Gastroenterological Surgery 2:406-412

28. Washington K, Watkins JR, Jay J, Jeyarajah DR (2019) Oncologic resection in laparoscopic versus robotic transhiatal esophagectomy. JSLS : Journal of the Society of Laparoendoscopic Surgeons 23(e2019):00017

29. Wang L, Yao L, Yan P et al (2018) Robotic versus laparoscopic Roux-en-Y gastric bypass for morbid obesity: a systematic review and meta-analysis. Obes Surg 28:3691-3700

30. Ilic D, Evans SM, Allan CA et al (2017) Laparoscopic and robotic-assisted versus open radical prostatectomy for the treatment of localised prostate cancer. Cochrane Database Syst Rev. https://doi.org/10.1002/14651858.CD009625.pub2

31. Jayne D, Pigazzi A, Marshall H et al (2017) Effect of roboticassisted vs conventional laparoscopic surgery on risk of conversion to open laparotomy among patients undergoing resection for rectal Cancer. JAMA 318:1569

32. Indes JE, Mandawat A, Tuggle CT et al (2010) Endovascular procedures for aorto-iliac occlusive disease are associated with superior short-term clinical and economic outcomes compared with open surgery in the inpatient population. J Vasc Surg 52:11731179.e1

33. Saliba W, Reddy VY, Wazni O et al (2008) Atrial fibrillation ablation using a robotic catheter remote control system. J Am Coll Cardiol 51:2407-2411

34. Kanagaratnam P, Koa-Wing M, Wallace DT et al (2008) Experience of robotic catheter ablation in humans using a novel remotely steerable catheter sheath. J Interv Card Electrophysiol 21:19-26

35. Beyar R, Gruberg L, Deleanu D et al (2006) Remote-control percutaneous coronary interventions. J Am Coll Cardiol 47:296-300

36. Weisz G, Metzger DC, Caputo RP et al (2013) Safety and feasibility of robotic percutaneous coronary intervention. J Am Coll Cardiol 61:1596-1600

37. Smilowitz NR, Balter S, Weisz G (2013) Occupational hazards of interventional cardiology. Cardiovasc Revasc Med 14:223-228

38. Andreassi MG, Piccaluga E, Gargani L et al (2015) Subclinical carotid atherosclerosis and early vascular aging from long-term Low-dose ionizing radiation exposure. Cardiovascular Interventions 8:616-627

39. Nogueira RG, Sachdeva R, Al-Bayati AR et al (2020) Robotic assisted carotid artery stenting for the treatment of symptomatic carotid disease: technical feasibility and preliminary results. Journal of NeuroInterventional Surgery 12:341-344

40. Bismuth J, Duran C, Stankovic M et al (2013) A first-in-man study of the role of flexible robotics in overcoming navigation challenges in the iliofemoral arteries. J Vasc Surg 57:14S-19S

41. Cochennec F, Kobeiter H, Gohel M et al (2015) Feasibility and safety of renal and visceral target vessel cannulation using robotically steerable catheters during complex endovascular aortic procedures. J Endovasc Ther 22:187-193

42. Mahmud E, Schmid F, Kalmar P et al (2016) Feasibility and safety of robotic peripheral vascular interventions. JACC: Cardiovascular Interventions 9:2058-2064

43. Perera AH, Riga CV, Monzon L et al (2017) Robotic arch catheter placement reduces cerebral embolization during thoracic 
endovascular aortic repair (TEVAR). Eur J Vasc Endovasc Surg 53:362-369

44. Mahmud E, Schmid F, Kalmar P et al (2020) Robotic peripheral vascular intervention with drug-coated balloons is feasible and reduces operator radiation exposure: results of the robotic-assisted peripheral intervention for peripheral artery disease (RAPID) study II. J Invasive Cardiol 32:380-384

45. Sajja KC, Sweid A, Al Saiegh F et al (2020) Endovascular robotic: feasibility and proof of principle for diagnostic cerebral angiography and carotid artery stenting. Journal of NeuroInterventional Surgery 12:345-349

46. Weinberg JH, Sweid A, Sajja K, et al (2020) Comparison of robotic-assisted carotid stenting and manual carotid stenting through the transradial approach. J Neurosurg 1-8

47. Desai VR, Lee JJ, Sample T et al (2021) First in Man pilot feasibility study in extracranial carotid robotic-assisted endovascular intervention. Neurosurgery 88:506-514

48. Jones B, Riga C, Bicknell C, Hamady M (2021) Robot-assisted carotid artery stenting: a safety and feasibility study. Cardiovasc Intervent Radiol 44:795-800

49. Riga CV, Cheshire NJW, Hamady MS, Bicknell CD (2010) The role of robotic endovascular catheters in fenestrated stent grafting. Journal of Vascular Surgery 51:810-820

50. Riga C, Bicknell C, Cheshire N, Hamady M (2009) Initial clinical application of a robotically steerable catheter system in endovascular aneurysm repair. J Endovasc Ther 16:149-153

51. Cheung S, Rahman R, Bicknell C et al (2020) Comparison of manual versus robot-assisted contralateral gate cannulation in patients undergoing endovascular aneurysm repair. Int J Comput Assist Radiol Surg 15:2071-2078

52. Riga CV, Bicknell CD, Rolls A et al (2013) Robot-assisted fenestrated endovascular aneurysm repair (FEVAR) using the
Magellan system. Journal of Vascular and Interventional Radiology 24:191-196

53. Behnamfar O, Pourdjabbar A, Yalvac E et al (2016) First case of robotic percutaneous vascular intervention for below-the-knee peripheral arterial disease. J Invasvie Cardiol 28:E128-E131

54. Riga CV, Bicknell CD, Sidhu R et al (2011) Advanced catheter technology: is this the answer to overcoming the long learning curve in complex endovascular procedures. Eur J Vasc Endovasc Surg 42:531-538

55. Marescaux J, Leroy J, Gagner M et al (2001) Transatlantic robotassisted telesurgery. Nature 413:379-380

56. Legeza P, Sconzert K, Sungur J et al (2021) Preclinical study testing feasibility and technical requirements for successful telerobotic long distance peripheral vascular intervention. The International Journal of Medical Robotics and Computer Assisted Surgery. https://doi.org/10.1002/rcs.2249

57. Madder RD, VanOosterhout S, Parker J, et al (2021) Robotic telestenting performance in transcontinental and regional pre-clinical models. Catheter Cardiovasc Intervent 97

58. Patel TM, Shah SC, Soni YY, et al (2020) Comparison of robotic percutaneous coronary intervention with traditional percutaneous coronary intervention. Circulation: Cardiovasc Intervent 13

59. George JC, Tabaza L, Janzer S (2020) Robotic-assisted balloon angioplasty and stent placement with distal embolic protection device for severe carotid artery stenosis in a high-risk surgical patient. Catheter Cardiovasc Interv 96:410-412

Publisher's note Springer Nature remains neutral with regard to jurisdictional claims in published maps and institutional affiliations. 